\title{
Tunneling in attosecond optical ionization and a dynamical time operator
}

\author{
M. Bauer \\ Instituto de Física \\ Universidad Nacional Autónoma de México \\ bauer@fisica.unam.mx
}

September 5, 2018

\begin{abstract}
The conundrum parameter-operator of time in quantum mechanics (QM), as well as the time-energy uncertainty relation and the tunneling delay time, have recently been addressed in attosecond optical ionization experiments. The parameter status of time in the time dependent Schrödinger equation (TDSE) is supported by the well-known Pauli's objection as well as by its interpretation as an emerging property of entanglement with a classical environment. On the other hand, the introduction of a self-adjoint dynamical time operator in Dirac's formulation of electron's relativistic quantum mechanics (RQM), yields an additional system observable that represents an internal time. In the present paper the relation of this internal time with the parametric (laboratory) time and its relevance to the tunneling measurements in these experiments is examined within the standard framework of RQM.

Keywords: tunneling photoionization; time operator; time-energy uncertainty relation; relativistic quantum mechanics
\end{abstract}

PACS: 03.65.-w ; 03.65.Ca ; 03.65.Pm; 32.80.Fb

\section{Introduction}

The conundrum parameter-operator of time in quantum mechanics (QM), as well as the time-energy uncertainty relation and the tunneling delay time, have recently been addressed again in the development of attosecond optical ionization experiments 1, 2]. The tunneling phenomenon, one of the earliest theoretical successes of QM, has been extensively debated in relation to the question of the time the particle spends in the barrier region. This has given rise to alternative definitions of tunneling times but has not been definitively resolved 3 , 4. On the other hand, the technical development of attosecond pulses of extreme ultaviolet radiation has allowed photionization processes where a tunneling delay 
time can be measured and compared to theoretical predictions, although using a time-energy uncertainty relation associated with the commutation relation rightfully objected by Pauli [3, 5, 6, 7].

Indeed the existence of a time-energy uncertainty relation analogue to the position-momentum one, conjectured by Heisenberg early on, faced from the start Pauli's objection to the existence of a time operator, to quote [8, p.63]: "...from the C.R. written above (cf. $[t, H]=i \hbar$ ) it follows that $H$ possesses continously all eigenvalues from $-\infty$ to $+\infty$, whereas on the other hand, discrete eigenvalues of $H$ can be present. We, therefore, conclude that the introduction of an operator $t$ is basically forbidden and the time $t$ must necessarily be considered as on ordinary number (" "number) in Quantum Mechanics". In the time dependent Schrödinger equation (TDSE) time appears as a parameter, not an operator [8, 9]. This led to a variety of alternative proposals for a time-energy uncertainty relation and an extensive discussion of time in quantum mechanics throughout several decades [10, 11, 12, 13, 14, 15. Pauli's argument, sustained also by the fact that the system's stability requires the energy to have a finite minimum, is still subject of current research, as well as the existence and meaning of a time-energy uncertainty relation [16, 17. The undisputed experimental corroboration of Schrödinger's equation supports the interpretation of the parameter $t$ as the laboratory time. Its presence in the dynamical evolution of microscopical systems (TDSE) has been atributed to the entanglement of these systems with a macroscopic classical environment 18 .

Recently however, it has been shown that Dirac's formulation of electron's relativistic quantum mechanics (RQM) does allow the introduction of a dynamical time operator that is self-adjoint [19]. Consequently, it can be considered an additional system observable representing an internal time, and proven to be subject to an uncertainty relation that circumvents Pauli's objection. In the present paper it is shown that it provides an equal footing of time and space in the analysis of the attosecond optical ionization processes, as suggested in Ref.61. These aspects are examined within the standard framework of RQM. The definition and main properties of the proposed time operator are recalled in Section 2. In Section 3 the ensuing time-energy uncertainty relation is derived. It is also compared in Appendix A to the Mandelstam-Tamm formulation extensively addressed in the discussion of tunneling. Section 4 develops its application to the attosecond optical ionization processes. Section 5 advances conclusions and possible developments.

\section{The dynamical time operator in RQM}

A dynamical self-adjoint "time operator"

$$
\hat{T}=\boldsymbol{\alpha} \cdot \hat{\mathbf{r}} / c+\beta \tau_{0}
$$

\footnotetext{
${ }^{1}$ In this respect, Dodonov's quoted paper (Ref.4 in Ref. 6), that claims that no unambigous and generally accepted results have been obtained so far, refers only to the present author paper of 1983 (Ann.Phys. 150, 1 (1983) ), but not to the 2014 paper 19] that introduces the dynamical time operator.
} 
has been introduced [19] in analogy to the Dirac free particle Hamiltonian $\hat{H}_{D}=$ $c \boldsymbol{\alpha} \cdot \hat{\mathbf{p}}+\beta m_{0} c^{2}$, where $\alpha_{i}(i=1,2,3)$ and $\beta$ are the $4 \times 4$ Dirac matrices, satisfying the anticonmutation relations:

$$
\alpha_{i} \alpha_{j}+\alpha_{j} \alpha_{i}=2 \delta_{i j} \quad \alpha_{i} \beta+\beta \alpha_{i}=0 \quad \beta^{2}=1
$$

$\tau_{0}$ represents in principle an internal property of the sysytem, to be determined. In the Heisenberg picture, using the relations 20, 21, 22]:

$$
\begin{gathered}
\boldsymbol{\alpha}(t)=\boldsymbol{\alpha}(0)+\left\{\boldsymbol{\alpha}(0)-c \hat{\mathbf{p}} / \hat{H}_{D}\right\}\left\{\exp \left(-2 i \hat{H}_{D} t / \hbar\right)-1\right\} \\
\beta(t)=\beta(0)+\left\{\beta(0)-m_{0} c^{2} / \hat{H}_{D}\right\}\left\{\exp \left(-2 i \hat{H}_{D} t / \hbar\right)-1\right\} \\
\hat{\mathbf{r}}(t)=\hat{\mathbf{r}}(0)+\left(c^{2} \hat{\mathbf{p}} / \hat{H}_{D}\right) t+i\left(c \hbar / 2 \hat{H}_{D}\right)\left\{\exp \left(-2 i \hat{H}_{D} t / \hbar\right)-1\right\}
\end{gathered}
$$

the time evolution of the time operator is given by:

$$
\begin{aligned}
\hat{T}(t) & =\boldsymbol{\alpha}(t) \cdot \hat{\mathbf{r}}(t) / c+\beta(t) \tau_{0}= \\
& =\boldsymbol{\alpha}(0) \cdot \hat{\mathbf{r}}(0) / c+\beta(0) \tau_{0}+\boldsymbol{\alpha}(0) \cdot\left(c^{2} \hat{\mathbf{p}} / \hat{H}_{D}\right) t+\text { oscillating terms } \\
& =\hat{T}(0)+\left(c \hat{\mathbf{p}} / \hat{H}_{D}\right)^{2} t+\text { oscillating terms }
\end{aligned}
$$

where use has been made of:

$$
c \boldsymbol{\alpha}(0) \cdot\left(c \hat{\mathbf{p}} / \hat{H}_{D}\right)=\left[\frac{d \hat{\mathbf{r}}}{d t}\right]_{t=0} \cdot\left(c \hat{\mathbf{p}} / \hat{H}_{D}\right)=\left(c \hat{\mathbf{p}} / \hat{H}_{D}\right)^{2}+\text { oscillating terms }
$$

Thus $\hat{T}(t)$ exhibits a linear dependence on $t$ with a superimposed oscillation (Zitterbewegung), as occurs with the time development of the position operator $\hat{\mathbf{r}}(t)$.

In this formulation, $\tau_{0}$ plays the role of an invariant quantity in the $(\boldsymbol{r}, \tau)$ space, i.e., $\tau_{0}^{2}=\tau^{2}-(\boldsymbol{r} / c)^{2}$, as $m_{0} c^{2}$ plays in the $(\boldsymbol{p}, E)$ space, namely $\left(m_{0} c^{2}\right)^{2}=$ $E^{2}-(c \boldsymbol{p})^{2}$. To mantain the fundamental indeterminacy modulo $n 2 \pi$ ( $n$ an integer) in the phase of the complex eigenfunctions one has to set, for $n=1$ :

$$
\tau_{0}=2 \pi \hbar /<\beta>\varepsilon=h / m_{0} c^{2}
$$

This is the de Broglie period 23, 24]. Together with the Compton wave length, it sets a unified spacetime Compton scale that limits the wave packets width in space and time before negative energy and negative time components (particle and antiparticle) occur significantly. Moreover, it supports the existence of an internal property, the de Broglie clock with a period $\tau_{0}=h / m_{0} c^{2}[25,26,27$.

It is now important to note that in the non relativistic energies case $\left\langle\hat{H}_{D}\right\rangle \simeq$ $m_{0} c^{2}$, one has, neglecting oscillating terms in Eq.6:

$$
\left\langle\hat{T}\left(t_{2}\right)\right\rangle-\left\langle\hat{T}\left(t_{1}\right)\right\rangle \simeq\left(v_{g p} / c\right)^{2}\left(t_{2}-t_{1}\right)
$$


Thus dynamical (internal) intervals are contracted with respect to parametric (external) intervals.

On the other hand, in the case of ultra relativistic energies, $\left\langle\hat{H}_{D}\right\rangle \simeq c p$ and Eq.6 yields:

$$
\left\langle\hat{T}\left(t_{2}\right)\right\rangle-\left\langle\hat{T}\left(t_{1}\right)\right\rangle \simeq\left\langle(c p / c p)^{2}\right\rangle\left(t_{2}-t_{1}\right)=t_{2}-t_{1}
$$

Dynamical (internal) intervals coincide with parametric (external) intervals.

Finnaly, the time operator, being self-adjoint, is the generator of continous momentum displacements, and thus indirectly of continous energy dispalcements within the positive and the negative energy branches, but not across the energy gap. In this way Pauli's objection is circumvented.

\section{The time-energy uncertainty relation}

The time operator and the Dirac Hamiltonian satisfy the commutaion relation [19]:

$$
\left[\hat{T}, \hat{H}_{D}\right]=i \hbar\{I+2 \beta K\}+2 \beta\left\{\tau_{0} \hat{H}_{D}-m_{0} c^{2} \hat{T}\right\}
$$

where $K=\beta\left(2 \mathbf{s} . \mathbf{l} / \hbar^{2}+1\right)$ is a constant of motion 20]. In the usual manner an uncertainty relation follows, namely:

$$
(\Delta T)\left(\Delta H_{D}\right) \geq(\hbar / 2)|1+2<\beta K>|=(3 \hbar / 2)\left|1+\frac{4}{3}\left\langle\mathbf{s} \cdot \mathbf{l} / \hbar^{2}\right\rangle\right|
$$

where $\Delta T=\sqrt{\left\langle\hat{T}^{2}\right\rangle-\langle\hat{T}\rangle^{2}}$ and $\Delta H=\sqrt{\left\langle\hat{H}_{D}^{2}\right\rangle-\left\langle\hat{H}_{D}\right\rangle^{2}}$.

To be noted is that the uncertainty of the present time operator is related to the uncertainty in position $\Delta \mathbf{r}$, in the same way as the energy uncertainty is related to the momentum uncertainty $\Delta \mathbf{p}$. Indeed:

$$
\begin{aligned}
(\Delta T)^{2} & =\left\langle\hat{T}^{2}\right\rangle-\langle\hat{T}\rangle^{2}=\left\langle\hat{\mathbf{r}}^{2} / c^{2}+\tau_{0}^{2}\right\rangle-\langle\hat{T}\rangle^{2}= \\
& \left.=\left\{(\Delta \mathbf{r})^{2}+\langle\hat{\mathbf{r}}\rangle^{2}\right\} / c^{2}+\tau_{0}^{2}-\left\{\langle\boldsymbol{\alpha} . \hat{\mathbf{r}}) / c+\beta \tau_{0}\right\rangle^{2}\right\}= \\
& \left.=(\Delta \mathbf{r})^{2} / c^{2}+\tau_{0}^{2}\left(1-\langle\beta\rangle^{2}\right)+\left(\langle\hat{\mathbf{r}}\rangle^{2}-\langle\boldsymbol{\alpha} . \hat{\mathbf{r}}\rangle^{2}\right) / c^{2}-2 \tau_{0}\langle\boldsymbol{\alpha} . \hat{\mathbf{r}}) / c\right\rangle\langle\beta\rangle
\end{aligned}
$$

Thus:

$$
\Delta T \gtrsim \Delta \mathbf{r} / c
$$

and similarly:

$$
\begin{aligned}
\left(\Delta H_{D}\right)^{2} & =\left\langle\hat{H}_{D}^{2}\right\rangle-\left\langle\hat{H}_{D}\right\rangle^{2}=c^{2}\left\langle\hat{\mathbf{p}}^{2}\right\rangle+\left(m_{0} c^{2}\right)^{2}-\left\langle\hat{H}_{D}\right\rangle^{2} \\
& =c^{2}\left\{(\Delta \mathbf{p})^{2}+\langle\hat{\mathbf{p}}\rangle^{2}\right\}+\left(m_{0} c^{2}\right)^{2}-\left\langle\hat{H}_{D}\right\rangle^{2} \gtrsim c^{2}(\Delta \mathbf{p})^{2}
\end{aligned}
$$

Then

$$
(\Delta T)\left(\Delta H_{D}\right) \gtrsim(\Delta \mathbf{r})(\Delta \mathbf{p}) \geq(3 \hbar / 2)
$$


The association of $\Delta T$ with $\Delta \mathbf{r}$, and of $\Delta H_{D}$ with $\Delta \mathbf{p}$, corresponds to Bohr's interpretation: the width of a wave packet, complementary to its momentum dispersion and thus to its energy dispersion, measures the uncertainty in the time of passage at a point of the trajectory.

In the presence of potentials dependent only on position, e.g., Coulomb type potentials, the above result is maintained as:

$$
\left[\hat{T}, \hat{H}_{D}+V(\hat{\mathbf{r}})\right]=\left[\hat{T}, \hat{H}_{D}\right]
$$

and the same uncertainty relation will follow.If in addition there is spherical symmetry, the initial position and momentum expectation values vanish, i.e. $\langle\mathbf{r}\rangle=0$ and $\langle\mathbf{p}\rangle=0$. Then Eqs.13 and 14 become:

$$
\begin{gathered}
(\Delta T)^{2}=(\Delta \mathbf{r})^{2} / c^{2}+\tau_{0}^{2}\left(1-\langle\beta\rangle^{2}\right) \geqq(\Delta \mathbf{r})^{2} / c^{2} \\
\left(\Delta H_{D}\right)^{2}=c^{2}(\Delta \mathbf{p})^{2}+\left(m_{0} c^{2}\right)^{2}\left(1-\langle\beta\rangle^{2}\right) \geqq c^{2}(\Delta \mathbf{p})^{2}
\end{gathered}
$$

\section{Tunneling time in attosecond optical ioniza- tion}

The sudden onset of a laser pulse opens the electron bound state at energy $E_{0}=-I_{p}$ to tunneling through a barrier created by an effective potential in the direction of the pulse polarization, modelled as [3, 7, 6]:

$$
V_{e f f}=-\frac{Z_{e f f} e}{|\mathbf{r}|}-\mathbf{F} \cdot \mathbf{r}
$$

The first term is the binding Coulomb potential and the second is the dipole interaction with a pulse of maximum intensity $F$. The barrier width $d_{B}$ in the radial direction, say $x$, of the electric field, is given by the difference between the entrance $x_{e,-}$ and exit $x_{e,+}$ points of the barrier (Fig.1 of Ref. ), i.e., the solutions to the equation:

$$
-\frac{Z_{e f f} e}{x}-F x=-I_{p}
$$

yielding:

$$
d_{B}(F) \doteq\left\{x_{e,+}-x_{e,-}\right\}=\left(I_{p} / F\right) \sqrt{1-4 Z_{e f f} e F / I_{p}^{2}}
$$

as given in Eq.13 of Ref.6.

As shown in Section 3 above, the minimum time uncertainty for spherical symmetry is:

$$
\Delta T=\Delta \mathbf{r} / c=\left\langle r^{2}\right\rangle^{1 / 2} / c
$$

where integration is carried over all directions. If now one assumes that the tunnelig internal time $\bar{\tau}_{T}$ in one direction is equal to $(1 / 4 \pi) \Delta T$ and that the 
time uncertainty is of the order of the time uncertainty associated with the barrier width $\left(\delta r=d_{B}(F)\right)$, one concludes that for a single direction the internal tunneling time is given by:

$$
\bar{\tau}_{T} \approx(1 / 4 \pi) d_{B}(F) / c
$$

i.e., $\bar{\tau}_{T}$ is proportional to the time it would take a photon to traverse the barrier width. Then from Eq.17, the laboratory tunneling time in the non relativistic regime is given by:

$$
\Upsilon_{T} \approx \bar{\tau}_{T} /\left(v_{g p} / c\right)^{2} \approx\left\{(1 / 4 \pi) d_{B}(F) / c\right\} /\left(v_{g p} / c\right)^{2} \gg \bar{\tau}_{T}
$$

There is thus a linear relation between laboratory tunneling time and barrier width, as has been experimentally obtained (Fig.3(d) of Ref.2).

The enhancing factor between internal and (laboratory) parameter times can be evaluated as follows. Ref.2 reports an electron tunneling time of 40 as for a barrier width of 13 a.u. $=6.88 \AA$. This gives a tunneling velocity $v_{g p}=$ $\frac{6.88}{40} \times 10^{10} \mathrm{~cm} / \mathrm{s}$, and a ratio $1 /\left(v_{g p} / \mathrm{c}\right)^{2}=304.22$. It follows then:

$$
\Upsilon_{T} \approx(1 / 4 \pi) 304.22\left[d_{B}(F) / c\right]
$$

For a barrier width of 20 a.u. $=10.58 \AA$ one obtains:

$$
\Upsilon_{T} \approx(1 / 4 \pi) 304.22\left[d_{B}(F) / c\right]=24.22 \times(10.58 / 3) \text { as }=85.4 \text { as }
$$

while for a barrier width of 8 a.u. $=4.233 \AA$ one obtains:

$$
\Upsilon_{T} \approx(1 / 4 \pi) 304.22\left[d_{B}(F) / c\right]=24.22 \times(4.233 / 3) \text { as }=34.2 \text { as }
$$

These value compare well with the experimental results shown in Fig.3(d) of Ref.2. The straight line joining these values has a slightly different slope of that of the FPI (Feynman Path Integral) quantum mechanical result, but falls within the experimental uncertainties.

The dependence on the field intensity is obtained using Eq.18, namely:

$$
\Upsilon_{T} \approx(1 / 4 \pi)\left[\left(I_{p} / F\right) \sqrt{1-4 Z_{e f f} e F / I_{p}^{2}}\right] / c\left(v_{g p} / c\right)^{2}
$$

which gives the observed shape of the dependence of the barrier width on the field intensity (Fig.3(b) of Ref.2).

\section{Conclusion}

The dynamical time operator provides a straightforward explanation within standard RQM of the tunneling times measured in the photoionization experiments. As an observable, it introduces an internal time in addition to the parameter (laboratory) time in the TDSE that has been shown to be an emergent property arising from the entanglement of a microscopic system with a 
classical environment in an overall closed time independent system, this property being apparent only to an internal observer [18, 28. There is no conumdrum parameter-operator of time in quantum mechanics, as both times are seen to play a role in RQM. Also predicted is an enhancement at low energies between internal and laboratory tunneling times that fits the measurements in attosecond optical ionization experiments.

Based on the position observable, the time operator is expected to exhibit a Zitterbewegung behaviour about its linear dependence on $t$. As occurs with the position one, its observation is beyond current technical possibilities. However it may be observable in systems that simulate Dirac's Hamiltonian, where position Zitterbewegung has allready been exhibited experimentally [29, 30, 31]. A corresponding time operator can be constructed in each case and perhaps its properties may be exhibited in similar experiments.

Finally, general relativity accords a dynamical behaviour to space-time, firmly confirmed recently by the detection of gravitational waves. As a dynamical time is definitively incompatible with a time parameter, this becomes from the start a fundamental "problem of time" in quantum gravity [32, 33, 34]. Whether the time operator here introduced has a relevance in this subject, is a venue to be considered [35].

\section{Appendix A: Mandelstam-Tamm time-energy uncertainty relation}

As an observable, the time operator can be subject to the Mandelstam-Tamm (MT) formulation of a time-energy uncertainty relation within standard QM[22, p.319], to wit: any observable $A$ represented by a self-adjoint operator $\hat{A}$ not explicitly dependent on time, satisfies the dynamical equation:

$$
(i \hbar) \frac{d}{d t}<\hat{A}>=<[\hat{A}, \hat{H}]>
$$

From the commutator $[\hat{A}, \hat{H}]$ it follows that the uncertainties defined $\Delta \hat{A}$ and $\Delta \hat{H}$ satisfy the relation:

$$
(\Delta \hat{A})(\Delta \hat{H}) \geq(1 / 2)|<[\hat{A}, \hat{H}]>|=(1 / 2)\left|\frac{d}{d t}<\hat{A}>\right|
$$

Then, associated to any system observable $\hat{A}$, a related time uncertainty is defined as:

$$
\Delta \hat{T}_{\hat{A}}^{m t}=\frac{\Delta \hat{A}}{\left|\frac{d}{d t}<\hat{A}>\right|}
$$

From Eqs. 17 and 18, it then follows that:

$$
\left(\Delta \hat{T}_{\hat{A}}^{m t}\right)(\Delta \hat{H}) \geq(\hbar / 2)
$$


This is the Mandelstam-Tamm time-energy uncertainty relation. $\Delta \hat{T}_{\hat{A}}^{m t}$ can be interpreted as "the time required for the center $\langle\hat{O}\rangle$ of this distribution to be displaced by an amount equal to its width $\Delta \hat{A} "[22]$.

Now let $\hat{A}$ be the dynamical time operator $\hat{T}=(\boldsymbol{\alpha} . \mathbf{r}) / c+\beta \tau_{0}$. Then, from Eq. 20 and Eq.12, one obtains:

$$
\Delta T_{\hat{T}}^{m t} \approx \frac{\Delta \hat{T}}{|\langle I+2 \beta K\rangle|}
$$

It follows that:

$$
\frac{\Delta \hat{T}}{|\langle I+2 \beta K\rangle|}\left(\Delta \hat{H}_{D}\right) \geq(\hbar / 2)
$$

or

$$
\begin{aligned}
(\Delta \hat{T})\left(\Delta \hat{H}_{D}\right) & \geq(\hbar / 2)|\langle I+2 \beta K\rangle|=(3 \hbar / 2)\left|1+\frac{4}{3}\left\langle\mathbf{s} \cdot \mathbf{l} / \hbar^{2}\right\rangle\right|= \\
& \left.=(3 \hbar / 2)\left|1+\frac{2}{3}\left\langle\left(\mathbf{j}^{2}-\mathbf{l}^{2}-\mathbf{s}^{2}\right) / \hbar^{2}\right\rangle\right| \geq 3 \hbar / 2\right)
\end{aligned}
$$

In the non relativistic limit $\left\langle\hat{H}_{D}\right\rangle \simeq m_{0} c^{2}$, neglecting the oscillating terms, Eq.3 yields:

$$
\langle\hat{T}(t)\rangle \simeq\langle\hat{T}(0)\rangle+\left\langle\left(c p / m_{0} c^{2}\right)^{2}\right\rangle t+\ldots=\langle\hat{T}(0)\rangle+\left(v_{g p} / c\right)^{2} t
$$

Thus:

$$
\frac{d<\hat{T}>}{d t}=\left\langle\left(c p / m_{0} c^{2}\right)^{2}\right\rangle=\left(v_{g p} / c\right)^{2}
$$

and

$$
\Delta T_{\hat{T}}^{m t} \simeq \frac{\Delta \hat{T}}{\left(v_{g p} / c\right)^{2}} \gg \Delta \hat{T}
$$

as $v_{g p}<<c$. The Mandelstam-Tamm uncertainty associated with the observable $\hat{T}$ overestimates largely the internal time standard uncertainty.

\section{References}

[1] Eckle, P. etal, "Attosecond Ionization and Tunneling Delay Time Measurements in Helium", Science 322, 1525 (2008)

[2] Landsman, A.S. et al., "Ultrafast resolution of tunneling delay time", Optica 1, $343(2014)$

[3] Orlando, G. et al., "Tunneling time, what does it mean?", J. Phys. B:At. Mol. Opt. Phys. 47, 204002 (2014)

[4] Landauer, r. and Ph. Martin, "Barrier interaction time in tunneling", Rev.Mod.Phys. 66. 217-228 (1994) 
[5] Maquet, M., J. Caillat and R. Taïeb, "Attosecond delays in photoionization: time and quantum mechanics", J.Phys. B: At.Mol.Opt.Phys. 47, 204004 (2014)

[6] Kullie, O., "Tunneling time in attosecond experiments and the time-energy uncertainty relation", Phys.Rev. A 92, 052118 (2015)

[7] Kullie, O., "Tunneling time in attosecond experiments, intinsic-type of time. Keldysh amd Mandelstam-Tamm time", J.Phys. B: At.Mol.Opt.Phys. 49, 095601 (2015)

[8] Pauli, W., "The general principles of quantum mechanics", SpringerVerlag, Berlin Heidelberg, footnote p.63 (1980)

[9] Dirac, P.A.M., "The principles of quantum mechanics" (4th ed.), Oxford, Clarendon Press (1958)

[10] Muga, J.G., R. Sala Mayato and I.L. Egusquiza , "Introduction", in J.G. Muga, R. Sala Mayato, I.L. Egusquiza (eds.) "Time in Quantum Mechanics", pp 1-28 Berlin Springer (2002); reprinted as "Time in Quantum Mechanics, Vol. 1", Lect. Notes Phys. 734, Springer-Verlag, Berlin (2008)

[11] Muga, J.G., A. Ruschhaupt and A. del Campo (eds), "Time in Quantum Mechanics, Vol. 2", Lect. Notes Phys. 789, Springer-Verlag, Berlin (2009).

[12] Bauer, M. and P.A. Mello, "The time-energy uncertainty relation", Ann.Phys. 111, 38-60 (1978)

[13] Busch, P., "The time-energy uncertainty relation", chapter 3 in J.G. Muga, R. Sala Mayato, I.L. Egusquiza (eds.) "Time in Quantum Mechanics", Berlin Springer (2002); revised version: arXiv:quant-ph/0105049v3 (2007)

[14] Briggs, J., "A derivation of the time-energy uncertainty relation", Journal of Physics: Conference Series 99, 012002 (2008)

[15] Hilgevoord, J., "Time in Quantum Mechanics", Am.J.Phys. 70, 301-306 (2002)

[16] Galapon, E.A., "Post-Pauli's Theorem Emerging Perspective on Time In Quantum Mechanics", Chapter 3 in Lect. Notes Phys. 789, 25-63 (2009)

[17] Boykin, T.B., N. Kharche and G. Klimeck, "Evolution time and energy uncertainty", Eur.J.Phys. 28, 673-678 (2007)

[18] Briggs, J.S. and Jan M. Rost, "Time dependence in quantum mechanics", Eur.Phys.J. 10, (2000); "On the Derivation of the Time-dependent Equation of Schrödinger", Found.Phys. 31, (2001)

[19] Bauer, M., "A dynamical time operator in Dirac's relativistic quantum mechanics", Int.J.Mod.Phys. A 29, 1450036 (2014) 
[20] Thaller, B., "The Dirac Equation", Springer-Velag, Berlin Heidelberg New York (1992)

[21] Greiner, W., "Relativistic Quantum Mechanics - Wave equations", (3 ${ }^{\mathrm{d}}$ ed.) Springer, Berlin Heidelberg New York (2000)

[22] Messiah, A., "Quantum Mechanics", Vol.I, p. 442, North-Holland Publishing Company, Amsterdam, and John Wiley\&Sons, New York London Sidney, 4th printing (1966)

[23] de Broglie, L., Ph.D. thesis; Ann. Phys.,Ser. 10e, t. III (1925). English translation reprinted in Ann.Fond.Louis de Broglie 17, 92 (1992)

[24] Baylis, W.E., "De Broglie waves as an effect of clock desynchronization", Can.J.Phys. 85, 1317-1323 (2007)

[25] Ferber, R., "A Missing Link: What is behind de Broglie's "periodic phenomenon"?, Found.Phys.Lett. 9, 575-586 (1996)

[26] Lan, S.Y. et al., "A Clock Directly Linking Time to a Particle Mass", Science 339, 554-557 (2013)

[27] Catillon, P. et al., "A Search for the de Broglie Particle Internal Clock by means of Electron Channeling", Found.Phys. 38, 659-664 (2008)

[28] Moreva, E. et al., "Time from quantum entanglement: an experimental illustration", arXiv:1310.4691v1 [quant-ph] (2013); "The time as an emergent property of quantum mechanics, a synthetic description of a first experimental approach", J.Phys.: Conference Series 626, 012019 (2015)

[29] Cserti, J. and G. David, "Unified description of Zitterbewegung for spintronic, graphene, and superconducting systems", Phys.Rev. B 74, 172305 (2006)

[30] Gerritsma, R. et al., "Quantum simulation of the Dirac equation", Nature 463, 68-71 (2010)

[31] LeBlanc, L.J. et al., "Direct observation of Zitterbewegung in a BoseEinstein condensate", New J.Phys. 15, 073011 (2013)

[32] Anderson, E., "The problem of time in quantum gravity", Ann.Phys. (Berlin) 524, 757-786 (2012) and references therein; also arXiv:1009.2157v3 [gr-qc]

[33] Isham, C.J., "Canonical Quantum Gravity and the Problem of Time", arXiv:gr-qc/9210011v1 (1992); "Prima Facie Questions in Quantum Gravity", arXiv:gr-qc/9310031v1 (1993)

[34] Butterfield, J. and C.J. Isham, "On the Emergence of Time in Quantum Gravity", arXiv:gr-qc/99010241 (1999) 
[35] Bauer, M., "Quantum Gravity and a Time Operator in Relativistic Quantum Mechanics", arXiv:gr-qc/1605.01659 (2016) 